Bio - grafía. Escritos sobre la Biología y su Enseñanza. ISSN 2027-1034

Edición Extraordinaria. p.p. 1255 - 1262

Memorias del IX Encuentro Nacional de Experiencias en Enseñanza de la Biología y la Educación Ambiental. IV Congreso Nacional de Investigación en Enseñanza de la Biología.

\title{
ENSEÑAR A PENSAR, CREAR, ACTUAR Y CONSTRUIR CRÍIICAMENTE: DESDE EL DESARROLLO DEL PENSAMIENTO CIENTÍFICO ESCOLAR Y LA ENSEÑANZA PARA LA COMPRENSIÓN' ${ }^{1}$.
}

\section{TEACHING TO THINK, CREATE, ACT AND CONSTRUCT CRTICALLY:FROM THE DEVELOPMENT OF THE SCIENTIFIC THINKING SCHOOL AND THE TEACHING FOR THE UNDERSTANDING}

\author{
García Vega Iris Beatriz ${ }^{2}$
}

\section{RESUMEN}

Esta investigación surge a partir del análisis crítico de la pertinencia y los retos actuales de la enseñanza y aprendizaje las ciencias naturales y la educación ambiental en Instituciones públicas como la Institución Educativa Municipal Cundinamarca de Zipaquirá (IEMCZ). El propósito gira entorno al desarrollo del pensamiento científico escolar en estudiantes de grado sexto y octavo, se busca la construcción de habilidades como el pensamiento crítico, el trabajo en equipo, la creatividad, el comportamiento autorregulado y la toma de decisiones que le permitan a los estudiantes apropiarse del conocimiento científico y tecnológico e interactuar con su realidad. La propuesta se materializa desde la Enseñanza para la Comprensión $(\mathrm{EpC})$, el uso de rutinas de pensamiento y de rúbricas de valoración continua que permiten orientar el proceso. Se enmarca en el enfoque cualitativo y el diseño de Investigación Acción pedagógica, metodología que favorece el involucrar a los estudiantes como agentes activos y el ejercicio reflexivo de la práctica pedagógica en la búsqueda de alternativas de solución. El Marco de la EpC, el uso de rutinas permiten superar la enseñanza defensiva y el miedo a perder el control, a darle importancia a la expectativas de los estudiantes, abrir espacio a sus preguntas y que estás sean el motor del aprendizaje en el aula.

PALABRAS CLAVES: Pensamiento científico escolar, EpC, rutinas de pensamiento

\section{ABSTRACT}

This research arises from the critical analysis of the pertinence and current challenges of teaching and learning of the Natural Sciences and Environmental Education in public institutions such as the Institución Educativa Municipal Cundinamarca de

\footnotetext{
${ }^{1}$ Este trabajo hace parte de la Investigación Pensar Crear y actuar las Bases para emprender desde el desarrollo del pensamiento Científico, lógico matemático y lógico lingüístico en estudiantes de básica secundaria de la IEM Cundinamarca en Zipaquirá, que se desarrolló como requisito para optar al título de Magister en Pedagogía de la Universidad de la Sabana (2017).

${ }^{2}$ Magister en Pedagogía de Universidad de La Sabana, Licenciada en Biología, por la Universidad Pedagógica Nacional, actualmente docente de Ciencias Naturales y Educación ambiental, en la Institución Educativa Municipal Cundinamarca de Zipaquirá.
} 
Bio - grafía. Escritos sobre la Biología y su Enseñanza. ISSN 2027-1034

Edición Extraordinaria. p.p. $1255-1262$

Memorias del IX Encuentro Nacional de Experiencias en Enseñanza de la Biología y la Educación Ambiental. IV Congreso Nacional de Investigación en Enseñanza de la Biología.

Zipaquirá (IEMCZ). The purpose revolves around the development of scientific school thinking in sixth and eighth grade, seeks to build skills such as critical thinking, teamwork, creativity, self-regulated behavior and decision making that allow Students to appropriate scientific and technological knowledge and interact with their reality. The proposal is materialized from the Teaching for Understanding $(\mathrm{EpC})$, the use of thought routines and continuous evaluation rubrics that guide the process. It is framed from the qualitative approach and the design of Research pedagogical action, methodology that favors to involve the students as active agents and the reflexive exercise of the pedagogical practice in the search of alternative solutions. The EpC Framework, the use of routines allow to overcome defensive teaching and the fear of losing control, to give importance to student expectations, to achieve the students make questions and these become in the engine of learning in the classroom.

KEYWORDS: school scientific thought, Teaching for Understanding, thought routines.

\section{INTRODUCCIÓN}

Enseñar a los estudiantes a pensar, crear y construir críticamente la realidad, conlleva a una continua reflexión sobre el rol que desempeñamos en los procesos de formación; ¿Somos transmisores de información u orientadores en la construcción de pensamiento científico?, ¿Generamos espacios abiertos a la discusión y al dialogo de saberes en nuestras clases o nos imponemos al creer ser los únicos dueños del conocimiento? ¿Son útiles en la cotidianidad de los estudiantes los contenidos que proponemos o solo sirven para llenar cuaderno?

Como lo plantean Claxton (1994), Meinardi (2010), Quitanilla (2012), Tamayo (2014), la enseñanza de las ciencias debe estar al alcance de todos los ciudadanos, debe fomentar la democracia, preparar a todos en una ciudadanía científicamente culta para que comprendan, interactúen y construyan el mundo en el que viven. Debe preparar para resolver problemas de su cotidianidad, generar un pensamiento científico y crítico que le permitan participar en la toma de decisiones en los asuntos públicos. Debe mostrar a las ciencias como una actividad humana y social, en la cual todos pueden participar. No es solo manejar la información, es construir las competencias que le permitan apropiarse del conocimiento científico y tecnológico e interactuar con su realidad. En este sentido, se consolida una visión que asume la enseñanza de las ciencias como una forma de pensar crear y actuar, que exige a docentes y estudiantes construir las competencias que le permiten identificar problemas, generar acciones oportunas, pertinentes, innovadoras y sostenibles que atiendan dichas necesidades (Escobedo 2009). Esta perspectiva se construye desde el fomento de habilidades como el pensamiento crítico, la creatividad, el trabajo en equipo, comportamiento autorregulado (Ver tabla 1): 
Bio - grafía. Escritos sobre la Biología y su Enseñanza. ISSN 2027-1034

Edición Extraordinaria. p.p. 1255 - 1262

Memorias del IX Encuentro Nacional de Experiencias en Enseñanza de la Biología y la Educación Ambiental. IV Congreso Nacional de Investigación en Enseñanza de la Biología.

Tabla 1 Habilidades del Pensamiento Científico.

\begin{tabular}{|c|l|}
\hline Creatividad & $\begin{array}{l}\text { Como lo plantean Sarmiento, (2010), Cerda (2010), Cardona y Araque, (2013), la creatividad es una actitud y } \\
\text { aptitud que tenemos todos los seres humanos sin importar la edad o el sexo, la cual facilita la resolución de } \\
\text { problemas, la toma de decisiones y el fluir de la originalidad y la innovación en lo que realizamos a diario. }\end{array}$ \\
\hline Pensamiento Crítico & $\begin{array}{l}\text { Está relacionado con la capacidad para resolver problemas, escuchar todas las partes de un conflicto, decidir } \\
\text { qué es lo pertinente y lo que no, ser capaz de resumir con claridad ideas complejas, dar explicaciones } \\
\text { coherentes y justificadas de cómo se llegó a determinada conclusión (Saiz, 2002; Facione, 2007; } \\
\text { Tamayo,2014). }\end{array}$ \\
\hline Proactividad & $\begin{array}{l}\text { Para autores como Covey (1989), Formichella (2004) las personas proactivas conocen a la perfección sus } \\
\text { valores y principios, toman decisiones conscientemente, crean oportunidades y las dirigen para generar } \\
\text { cambios. }\end{array}$ \\
\hline Toma de decisiones & $\begin{array}{l}\text { Está relacionada con la capacidad para asumir riesgos e identificar aquellos inconvenientes que pueden afectar } \\
\text { el desarrollo de sus actividades y actuar oportunamente para poder controlarlos y reducirlos (Marina 2005, } \\
\text { Orrego 2008). }\end{array}$ \\
\hline Trabajo en equipo & $\begin{array}{l}\text { Es el esfuerzo mancomunado que realizan dos o más personas en consecución de un objetivo determinado, } \\
\text { para ello es necesario la cooperación, la disposición, la sinergia, la disciplina y la responsabilidad (Buchloz y } \\
\text { Roth, 1992 y Formichella 2004). }\end{array}$ \\
\hline autorregulado & $\begin{array}{l}\text { Para autores como (Pintrich 2000, Baumeister 2004, Formichella 2004) es una característica emprendedora } \\
\text { que se relaciona directamente con la capacidad que tiene el sujeto de integrar los conocimientos, las emociones } \\
\text { y las percepciones, de tal manera que en el contexto de sus relaciones o procesos cognitivos regule su } \\
\text { comportamiento y le permita persistir en buenas tareas. }\end{array}$ \\
\hline
\end{tabular}

Así, el pensamiento científico escolar va más allá de aprender o memorizar contenidos requiere una formación integral que permita desarrollar en contexto estas habilidades.

\section{Enseñanza para la Compresión y Desarrollo del Pensamiento Científico Escolar}

El marco de la Enseñanza para la Compresión y la estrategia de hacer visible el pensamiento por medio de las rutinas (Perkins, 1997; Wiske, 1999; Richhart, 2014), se acoge como una valiosa herramienta para desarrollar el pensamiento científico escolar. Esta se sustenta en Tópicos generativos, Metas de comprensión, Desempeños de comprensión, y la Evaluación (2004). En la investigación se diseñaron tres unidades de comprensión tituladas ¿Cómo pueden hacer los fósiles de dinosaurios para que los carros funcionen? Orienta a los estudiantes en comprender cómo a partir de los derivados del petróleo se produce energía y el impacto ambiental de su uso. ¿Qué pasa debajo de la costra?, favorece la comprensión de la célula como la unidad estructural de los seres vivos. Y ¿Qué le pasa a tu cuerpo cuando te enamoras? que permite a los estudiantes comprender la reproducción humana y la toma de decisiones en la construcción de un proyecto de vida.

Las rutinas de pensamiento, orientan el aprendizaje de los estudiantes y dan estructura a las discusiones del aula, permiten explorar ideas, relacionarlas con otras, sintetizarlas, organizarlas y profundizar sobre ellas. Estas favorecen por ejemplo, que a partir de una imagen (veo-pienso-me pregunto) se favorezca la observación, interpretación y generación de preguntas, los estudiantes pueden de forma individual y grupal hacer descripciones, construir explicaciones e interpretaciones, tener en cuenta diferentes puntos de vista, preguntarse, establecer conexiones, entre otros, a su vez implican ser creativos (curiosidad, fluidez), críticos (identificar, analizar), autorregulados (manejo de las emociones, organización de tiempos y tareas), proactivos (iniciativa, perseverancia), tomar decisiones (solución de problemas), trabajar en equipo y ser líderes (disposición y responsabilidad) 
Bio - grafía. Escritos sobre la Biología y su Enseñanza. ISSN 2027-1034

Edición Extraordinaria. p.p. 1255 - 1262

Memorias del IX Encuentro Nacional de Experiencias en Enseñanza de la Biología y la Educación Ambiental. IV Congreso Nacional de Investigación en Enseñanza de la Biología.

El pensamiento es un concepto complejo, está relacionado con la actividad intelectual que nos permite resolver problemas, tomar decisiones, observar, establecer hipótesis, hacer preguntas, aprender, actuar en otros contextos con los referentes construidos Richhart, (2014). Por lo tanto, desde la enseñanza de las ciencias naturales es de vital importancia reconocerlo, y hacerlo visible en el aula, para ofrecerle a los estudiantes más oportunidades donde construir y aprender significativamente.

\section{METODOLOGÍA}

El trabajo se adelanta en la Institución Educativa Municipal Cundinamarca, en el municipio de Zipaquirá (IEMCZ), con estudiantes de grado sexto y octavo de educación básica secundaria, de estratos uno y dos, con edades que oscilan entre los 11 y los 16 años. Participaron 6 cursos de 40 estudiantes. El centro educativo se encuentra ubicado en una zona vulnerable del municipio, con influencia de bandas organizadas dedicadas al microtráfico.

Esta investigación se aborda desde un enfoque cualitativo y un diseño de investigación acción pedagógica con un alcance interpretativo y propositivo siguiendo los lineamientos de Bonilla (2005) y Hernández Sampieri (2010). Se emplearon instrumentos como, Rutinas de pensamiento, Rúbricas de valoración y Diarios de campo. Además, se diseñaron y desarrollaron tres unidades de comprensión para el fomento de las habilidades del pensamiento científico escolar de los estudiantes, las cuales se valoran con rúbricas de evaluación diseñadas en la investigación. Las categorías surgen de las habilidades del pensamiento científico, la enseñanza, el aprendizaje y pensamiento.

Tabla 2 Habilidades del Pensamiento Científico Escolar

\begin{tabular}{|c|c|c|}
\hline Habilidad/Criterios & NIVEL INICIAL $6^{\circ}$ Y $70^{\circ}$ & NIVEL MEDIO $8^{\circ}$ Y $9^{\circ}$ \\
\hline $\begin{array}{l}\text { Pensamiento Crítico. } \\
\text { (Identifica problemas, Reconace } \\
\text { información relevante, identifica } \\
\text { conceptos e ideas, subdivide } \\
\text { problemas, establece conexiones). }\end{array}$ & $\begin{array}{l}\text { Interpreta y evalúa } \\
\text { situaciones concretas y } \\
\text { cotidianas en la resolución } \\
\text { de problemas }\end{array}$ & $\begin{array}{l}\text { Infiere y explica } \\
\text { situaciones abstractas } \\
\text { para proponer soluciones } \\
\text { y trasladarlas a otros } \\
\text { contextos. }\end{array}$ \\
\hline $\begin{array}{l}\text { Pensamiento Creativo. (Curiosidad } \\
\text { y Motivación, fluidez, flexibilidad y } \\
\text { divergencia, innovación y } \\
\text { originalidad). }\end{array}$ & $\begin{array}{l}\text { Utilizar la curiosidad para } \\
\text { generar fluidez y proponer } \\
\text { ideas diversas. }\end{array}$ & $\begin{array}{l}\text { Evaluar y argumentar } \\
\text { ideas viables que } \\
\text { generen cambio y } \\
\text { novedad. }\end{array}$ \\
\hline $\begin{array}{l}\text { Trabajo en equipo. } \\
\text { (Cooperación, disposi ción, } \\
\text { responsabilidad y resolución de } \\
\text { conflictos). }\end{array}$ & $\begin{array}{l}\text { Escuchar y expresar ideas } \\
\text { siguiendo el protocolo } \\
\text { establecido. }\end{array}$ & $\begin{array}{l}\text { Cooperar para lograr una } \\
\text { meta común o tomar } \\
\text { decisiones en conjunto. }\end{array}$ \\
\hline $\begin{array}{l}\text { Proactivid ad. } \\
\text { (Iniciativa, perseverancia, } \\
\text { adaptación y planificación). }\end{array}$ & $\begin{array}{l}\text { Visualizar experiencias y } 1 \text { a } \\
\text { realidad presente. }\end{array}$ & $\begin{array}{l}\text { Examinar alternativas de } \\
\text { solución y decidir } \\
\text { independientemente a la } \\
\text { influencia externa. }\end{array}$ \\
\hline $\begin{array}{c}\text { Comportamien to Autorregulado } \\
\text { (Conacimiento de capacidades, } \\
\text { manejo de emaciones, organización } \\
\text { de tiempos y tareas, desarrollo de } \\
\text { planes de mejora personal y } \\
\text { autoevaluación). }\end{array}$ & $\begin{array}{l}\text { Valorar una tarea a realizar } \\
\text { considerando metas y } \\
\text { restricciones. }\end{array}$ & $\begin{array}{l}\text { Evaluar su propio } \\
\text { conocimiento y } \\
\text { habilidades, visualizando } \\
\text { sus fortalezas y } \\
\text { debilidades. }\end{array}$ \\
\hline $\begin{array}{c}\text { Toma de decisiones } \\
\text { (Establece } \\
\text { Criterias y alternativas de solución } \\
\text { frente all riesgo, Afronta problemas y } \\
\text { aprende de los errores). }\end{array}$ & $\begin{array}{l}\text { Establece criterios y } \\
\text { alternativas solución a un } \\
\text { problema o situación. }\end{array}$ & $\begin{array}{l}\text { Propone y lleva a otros } \\
\text { contextos la toma de } \\
\text { decisiones con base a su } \\
\text { proyecto de vida. }\end{array}$ \\
\hline
\end{tabular}


Bio - grafía. Escritos sobre la Biología y su Enseñanza. ISSN 2027-1034

Edición Extraordinaria. p.p. 1255 - 1262

Memorias del IX Encuentro Nacional de Experiencias en Enseñanza de la Biología y la Educación Ambiental. IV Congreso Nacional de Investigación en Enseñanza de la Biología.

\section{RESULTADOS Y ANÁLISIS}

A partir de la implementación de las Unidades de compresión, de los registros, análisis de los diarios de campo, fotografías, cuadernos y proyectos de síntesis de los estudiantes, se establecen niveles altos (mayores al 70\%) de desempeño en cada una de las habilidades y los siguientes resultados generales:

Figura 1. Nivel de desarrollo de las Habilidades del Pensamiento Científico para el grado Octavo y Sexto

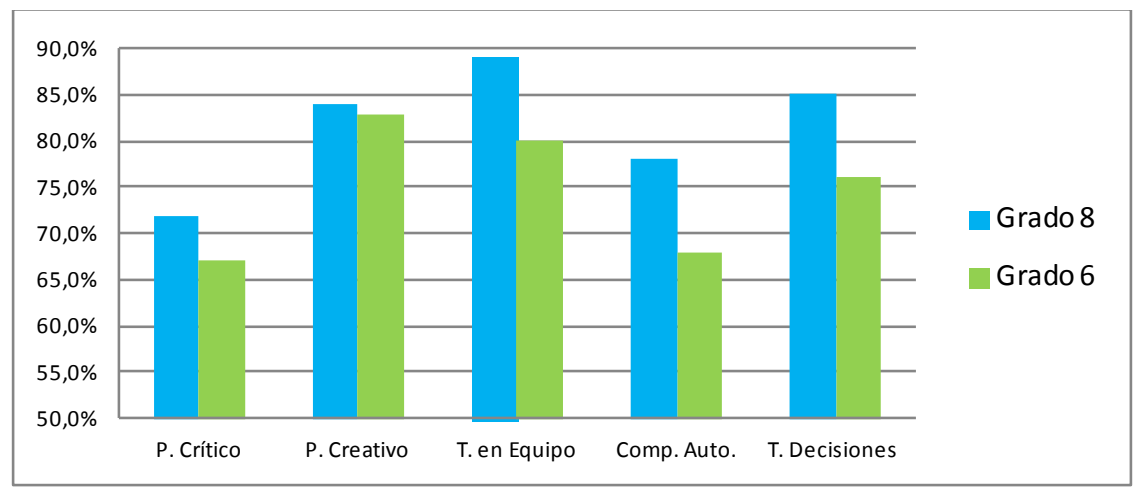

Creatividad: En grado sexto, esta habilidad logra los mayores niveles de desempeño (ver figura1), los estudiantes se caracterizan por plantear gran diversidad de preguntas, novedosas y llamativas, estas se convierten en el motor de las actividades propuestas, y permiten que el trabajo sea más significativo. Los estudiantes de grado octavo se interesan por plantearse diversas preguntas, por las preguntas de otros y colaborar en la búsqueda de respuestas, sus actividades presentas alternativas novedosas y eficaces que dan solución a las situaciones planteadas.

Trabajo en Equipo: Como lo muestra la figura 1 en esta habilidad los estudiantes de octavo logran sus mejores desempeños. Para los primeros, la cooperación, disposición hacia el trabajo, responsabilidad, y resolución de conflictos se llevan a la práctica durante las actividades de síntesis en la elaboración conjunta de respuestas a las preguntas orientadoras. En ella los estudiantes son miembros activos, que asumen el liderazgo, se esfuerzan en el grupo por alcanzar la meta propuesta, se reconocen los aportes de todos los integrantes de los equipos, especialmente el de Juliana Flores estudiante no oyente que por primera vez se motiva y participan activamente en la socialización del trabajo realizado. Para los segundos en situaciones de conflicto proponen alternativas para el consenso con la ayuda de un mediador

El comportamiento autorregulado y la toma de decisiones de manera progresiva los estudiantes logran reconocer sus capacidades intelectuales, emocionales, físicas, la importancia de construir una vida emocional equilibrada manifestando tolerancia, empatía, solidaridad, frente al reconocimiento de su sexualidad. En sexto grado los estudiantes presentan dificultades para controlar emociones primarias, para la organización de tiempos y tareas, siempre pospone el trabajo hasta el límite.

Frente al Pensamiento crítico es la habilidad que muestra mayores dificultades los estudiantes se destacan por explicar las situaciones propuestas en los hilos conductores, diseñar soluciones, y en algunos casos trasladarlas a otros contextos. 
Bio - grafía. Escritos sobre la Biología y su Enseñanza. ISSN 2027-1034

Edición Extraordinaria. p.p. $1255-1262$

Memorias del IX Encuentro Nacional de Experiencias en Enseñanza de la Biología y la Educación Ambiental. IV Congreso Nacional de Investigación en Enseñanza de la Biología.

Para el grado sexto, se reconocen las situaciones problemicas propuestas, se motivan al construir explicaciones a situaciones cercanas, les cuesta reconocer la información relevante de la irrelevante, los conceptos e ideas claves y establecimiento de conexiones en la realización de mapas y esquemas.

\section{Discusión}

El marco de la EpC, hacer visible el pensamiento, por medio de rutinas constituyen un insumo novedoso para el desarrollo del pensamiento científico escolar y las competencias propuestas por el MEN. Durante el periodo de implementación de la investigación los resultados académicos de los estudiantes, reportados en la plataforma de Cuidad Educativa, evidencian el logro y la mejora significativa de los aprendizajes propuestos por el área. Esta estrategia permite formar ciudadanos críticos, creativos, proactivos, que tengan un comportamiento autorregulado, analicen los riesgos al tomar decisiones, sean líderes capaces de trabajar en equipo en la búsqueda del bien común o de metas trazadas por una comunidad y desde allí transformar su realidad.

El trabajo consolida como elementos estructurantes del quehacer docente el cuestionar, escuchar y documentar las ideas de los estudiantes. Se cuestiona a través de preguntas constructoras y las situaciones problémicas propuestas que invitan a los estudiantes a la aplicación, el análisis, la interpretación, el establecimiento de conexiones, permite guiar las ideas y conceptos. Escuchar es la base para comunicarnos y construir aprendizaje, escuchar con respeto y valorar las ideas de los estudiantes muestra interés por su pensamiento y le da el valor que merece en clase. La documentación se realiza a través de dibujos, escritos, fotos, y mapas, elaborados por los estudiantes y los registros de las rutinas de pensamiento que permiten evidenciar como ocurre el aprendizaje, hacer el seguimiento a los cambios en las ideas y explicaciones que construyen los estudiantes y realizar el seguimiento a la propuesta de unidades diseñadas.

El uso de rutinas permite visibilizar el pensamiento de los estudiantes y hacer de él motor de desarrollo en las clases estas favorece los movimientos de pensamiento y la construcción de habilidades del pensamiento científico escolar. Promueven la participación activa, los empodera, les ayuda a reconocer sus habilidades y a adquirir autonomía. A la par, las formas de trabajo en el aula, bien sean individuales o grupales, ahora están enfocadas a promover el pensamiento y el aprendizaje, requerimientos indispensables para la comprensión.

La evaluación en el marco de la EPC, reúne una serie de elementos como los plantea Morales y Restrepo (2015), al afirmar que posee las características de ser formativa, al proveer la identificación de aspectos por mejorar en las dos partes implicadas; es una fuente de motivación hacia las metas de comprensión, que compromete a los estudiantes en sus procesos de aprendizaje y por tanto estimula la metacognición, a través de la reflexión constante.

\section{CONCLUSIONES}

La formación de la Maestría y el trabajo de investigación acción en el marco de la Enseñanza para la Compresión, el uso de las rutinas de pensamiento ha permitido el enriquecer mi quehacer como maestra, el desarrollo de habilidades del 
Bio - grafía. Escritos sobre la Biología y su Enseñanza. ISSN 2027-1034

Edición Extraordinaria. p.p. 1255 - 1262

Memorias del IX Encuentro Nacional de Experiencias en Enseñanza de la Biología y la Educación Ambiental. IV Congreso Nacional de Investigación en Enseñanza de la Biología.

pensamiento científico escolar, ha generado otras manera orientar la enseñanza y el aprendizaje de las ciencias en mi contexto. Dicho de otra forma, como docentes estamos invitados y motivados a pensar en los estudiantes al diseñar la gestión de la clase en ¿Qué espero que comprendan? ¿Cómo llegan a comprender? ¿Para qué comprender determinado tópico? $Y$ ¿cuál será esa mejor forma de comunicar a los demás el nivel de comprensión? De igual manera este proceso de formación personal como docentes es una invitación a registrar y adelantar investigaciones en el aula, para compartir experiencias y abrir discusiones en torno al conocimiento disciplinar, pedagógico, didáctico y contextual del profesor de cualquier asignatura, la $\mathrm{EpC}$, las rutinas, y hacer visible el pensamiento son herramientas poderosas para que estudiantes y docentes logren pensar, crear y actuar con flexibilidad a partir de lo que saben en diferentes contextos.

\section{REFERENCIAS}

- Bonilla, E., \& Rodríguez, P. (2005). Más allá de los métodos. Investigación en Ciencias Sociales.

- Bernal, C. (2010). Metodología de la investigación. (3ª ed.). Colombia: Pearson Educación.

- Buchloz, R. (1992). Wilson Learning Corp. Cómo crear un equipo de alto rendimiento en su empresa. Buenos Aires: Atlántida.

- Cerda, H. (2011). La creatividad en la ciencia y en la educación. COOP. EDITORIAL MAGISTERIO. Tercera edición

- Claxton, G., \& Barberán, G. S. (1994). Educar mentes curiosas: el reto de la ciencia en la escuela. Visor.

- Elliott, J. (1993). El cambio educativo desde la investigación-acción. Ediciones Morata.

- Escobedo, H., Jaramillo, R., \& Bermúdez, Á. (2004). Enseñanza para la comprensión. Educere, 8(27), 529-534.

- Facione, P. (2007). Pensamiento Crítico: ¿Qué es y por qué es importante?. Versión en Español: http://www.eduteka.org/PensamientoCriticoFacione.php; Última revisión septiembre, 01 de 2015.

- Gatica-Lara, F., \& Uribarren-Berrueta, T. D. N. J. (2013). ¿ Cómo elaborar una rúbrica?. Investigación en educación médica, 2(5), 61-65.

- Hernández Sampieri, R., Fernández Collado, C., \& Baptista Lucio, P. (2010). Metodología de la investigación. México: Editorial Mc Graw Hill.

- Kemmis, S., \& Taggart, M. C. Robin, 1984, Cómo Planificar la Investigación Acción. Barcelona, Laertes.

- López, Y. (2013). Leer para comprender, escribir para transformar. Mineducación.

- Meinardi, E. y otros (2010). Educar en ciencias. Paidós. 
Bio - grafía. Escritos sobre la Biología y su Enseñanza. ISSN 2027-1034

Edición Extraordinaria. p.p. 1255 - 1262

Memorias del IX Encuentro Nacional de Experiencias en Enseñanza de la Biología y

la Educación Ambiental. IV Congreso Nacional de Investigación en Enseñanza de la Biología.

- Morales Benitez, M. Y. (2015). La visibilizarían del pensamiento, una herramienta esencial en la evaluación para el aprendizaje (Doctoral dissertation).

- Stone Wiske, M. (1999). La enseñanza para la comprensión. Vinculación entre la investigación y la práctica. Edit Paidos,

- Orrego, C. I. (2008). La dimensión humana del emprendimiento. Revista Ciencias Estratégicas, 16(20), 225-235. Medellin, Colombia.

- Printrich,2000. El aprendizaje autorregulado como medio y meta de la educación. Papeles del psicólogo, 27(3).

- Quintañilla, M. y otros (2012). Historia y Filosofía de la Ciencias, Aportes para una nueva aula de ciencias, promotora de ciudadanía y valores. Alambique: Didáctica de las Ciencias Experimentales.

- Ritchhart, R. (2014). Hacer visible el pensamiento: cómo promover el compromiso, la comprensión y la autonom ía de los estudiantes. Paidós.

- Saiz, C. y Nieto, A. M. (2002). Pensamiento crítico: capacidades y desarrollo. En C. Saiz (Ed.), Pensamiento Crítico: Conceptos Básicos Y Actividades Prácticas. Madrid: Pirámide.15-19.

- Stone Wiske, M. (1999). La enseñanza para la comprensión. Vinculación entre la investigación y la práctica. Edit Paidos,

- Tamayo, O. y otros (2014). Pensamiento Crítico en el Aula de Ciencias. Cap.1 El pensamiento Crítico en la Educación. Universidad de Caldas.23-40.

- Wiske, M. S. (1999). Enseñanza Para La Comprensión, La. Paidc"s. 